\title{
METHOD FOR SUPERVISORY IMPLEMENTATION OF MANIPULATION OPERATIONS BY UNDERWATER VEHICLES
}

\author{
Vladimir Filaretov, Alexander Konoplin, Nikita Konoplin \& Bidyadhar Subudhi
}
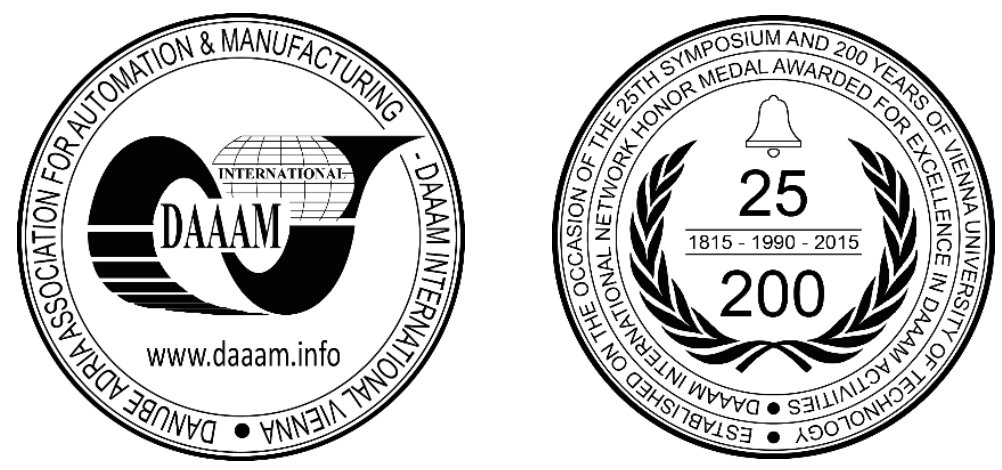

This Publication has to be referred as: Filaretov, V[ladimir] F[edorovich]; Konoplin, A[lexander]; Konoplin, N[ikita] \& Subudhi, B[idyadhar] (2017). Method for Supervisory Implementation of Manipulation Operations by Underwater Vehicles, Proceedings of the 28th DAAAM International Symposium, pp.0506-0512, B. Katalinic (Ed.), Published by DAAAM International, ISBN 978-3-902734-11-2, ISSN 1726-9679, Vienna, Austria

DOI: $10.2507 / 28$ th.daaam.proceedings.071

\begin{abstract}
At present, the underwater vehicles equipped with multilink manipulators are used to perform a wide range of survey, technological and research operations in the depths of the world ocean. But in most cases, said manipulation operations are performed with help of specially trained operators. Based on their experience and knowledge, these operators plan the trajectories of working tools of underwater manipulators. However, it is very difficult to quickly and accurately solve manipulation tasks, identifying the location of the object of work with help of video images. This leads to a decrease in the productivity and an increase in the probability of errors. The work proposes the new method for the supervisory implementation of manipulative operations by means of multilink manipulators mounted on underwater vehicles. This method involves the construction of mathematical models of objects of work with the help of on-board sonars. Herewith, the formation of target points and spatial trajectories of the manipulator's working tools is carried out by means of the targeting of the optical axis of the camera taking into account the requirements for the implementation of specific technological operations.
\end{abstract}

Keywords: supervisory implementation; underwater vehicle; multilink manipulator; underwater operations; spatial trajectory; control system

\section{Introduction}

The Analysis of current trends in the design and operation of underwater vehicles (UV) equipped with multi-link underwater manipulators (MMs) shows that the functionality of these vehicles is expanded due to the creation of the highquality navigation systems and devices [1]. In addition, the analysis shows that new methods for the environment recognition and the motion trajectories planning of the UVs and their MMs [2,3] also are created. At the same time, the majority of the underwater technological and research manipulation operations are still performed in a manual mode by the specially trained operators of the UVs. These operators basing on their experience and knowledge plan the trajectories of the movement of the MM working tools. However, the quickly and accurately performing of the manipulation operations without direct contact with the working object and with the information about its location from the video image only is very difficult for the operator. This leads to the performance decreasing of his work and to the probability increasing of the errors. 
In particular, the indicated problem was confirmed in the deep-sea scientific research expeditions in the Japan, Bering and Okhotsk Seas when we have worked with the UV Sub-Atlantic Comanche 18 equipped with the Schilling Orion 7P MM. The manual taking of samples of the soil, the geological rocks and the bacterial mats (see Fig. 1) leads to the fast tiring of operators, to the significant increasing in the time of the operations performing, to the errors appearing in work and even to the working tools damage.

To solve this problem the work proposed and considered a new method of supervisory performing of manipulation operations using the MM installed on the UV.

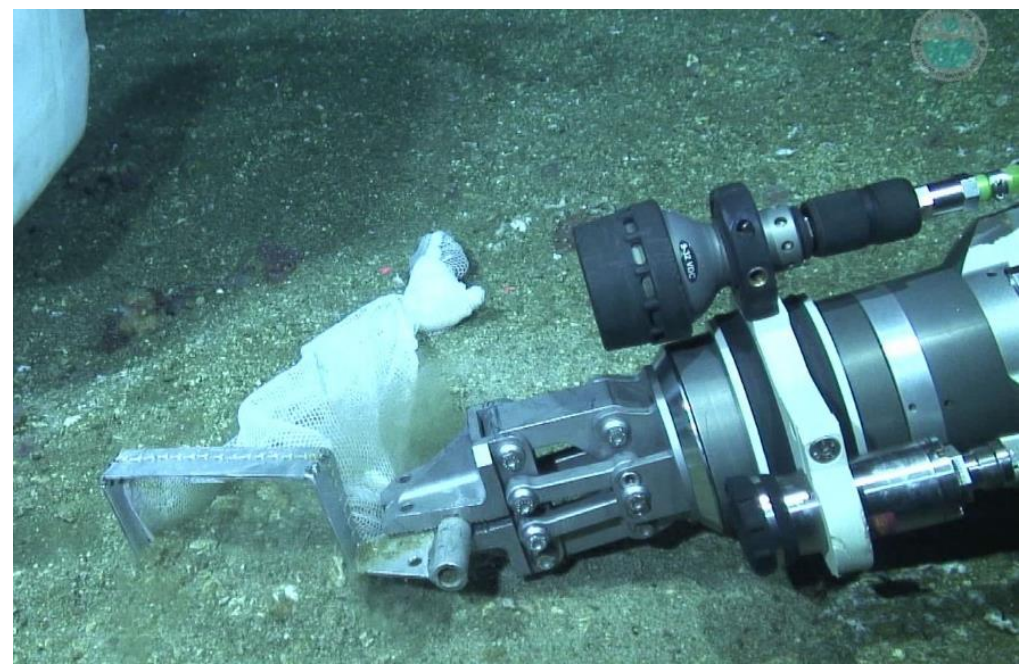

Fig. 1. The performing of the manipulation operation by the Schilling Orion 7P MM

\section{Description of the method of supervisory control of the MM installed on the UV}

The proposed method firstly defines the geometric shape and the location of the objects of work with the help of the onboard sonars. Then the operator directs the camera installed on the UV to the working object and specifies one or more operating points belonging to this object. The camera can change the spatial orientation of its optical axis. The spatial trajectory of motion and the orientation of the MM working tool are automatically formed from the coordinates of the given points belonging to the surface of the working object and depends on the manipulation task.

The implementation of the supervisory control of a soil sampling box on the example of the taking of a surface layer of soil is shown in Fig. 2. This box is moved by the MM 1 installed on the UV 2. The point cloud is formed via the multibeam hydroacoustic sonar 3 in a real time. The mathematical model 4 of the bottom surface is formed using the point cloud. This model is used to the further trajectory formation of the MM working tool. In particular, for the soil selection in the places of accumulation of bacterial mats, the operator specifies the $s$ target points $A_{i}(i=\overline{1, s})$ via the moving of the optical axis 5 of the camera. These points are the intersection points of this axis with the bottom surface. The trajectory 6 of the movement of the MM working tool 2 is formed at the given target points. When the taking of surface layer of soil is performing, the vector $\vec{a}$ of the soil sampling box must always be perpendicular to the bottom surface, and the vector $\vec{n}$ must be directed to the next target point $A_{i+l}$, while the MM moves along the trajectory (see Fig. 2). Furthermore, the vector $\vec{n}$ is perpendicular to the vector $\vec{a}$.

In many cases, the surface of the bottom without significant unevenness can be represented in the form of an averaged plane [4]. This plane can be described by the equation in the normal form [5] in the rectangular coordinate system (CS) $x y z$ rigidly connected to the UV body:

$$
a x+b y+c z=d,
$$

where $x, y$ and $z$ are the axes of the connected CS. The origin of this CS is located in the UV center of buoyancy $O, x$ axis coincides with the horizontal-longitudinal axis of the UV, $z$ axis coincides with the vertical axis of the UV, $y$ axis is righthanded. $a, b$ and $c$ are the direction cosines of the unit vector $\vec{m}=[a, b, c]$ of the normal to the plane (1), which coincides with the vector $\vec{a}$. The value $d$ is the distance from the origin of the CS $x y z$ to this plane. The elements of vector $\vec{m}$ and point $d$ are calculated using the formed cloud of points belonging to the bottom surface, according to the method described in [6].

The coordinates of the target points $A_{i}$ (see Fig. 2) must be calculated in the CS $x y z$ for the forming of the MM working tool motion trajectories. If the camera is directed to the point $A_{i}$, then the position of this camera is determined in the CS $x y z$ by the predetermined point $P_{i}=\left[P_{i x}, P_{i y}, P_{i z}\right]$ located on the optical axis. The orientation of the optical axis of the 
camera is determined by the unit vector $\vec{d}_{i}=\left[d_{i x}, d_{i y}, d_{i z}\right]$. In this case, the optical axis of the camera, which coincides with the vector $\vec{d}_{i}$, emerges from the point $P_{i}$ to the direction of the bottom plane (1) and described by the equation:

$$
A_{i}=P_{i}+t_{i} \vec{d}_{i}
$$

where $t_{i}$ is the parameter varies within range $[0, \infty)$. The value of the parameter $t_{i}$ at the point of intersection of the axis (2) with the plane (1) can be obtained by the substitution of the equation (2) in (1) [7]:

$$
\begin{aligned}
& a\left(P_{i x}+t_{i} d_{i x}\right)+b\left(P_{i y}+t_{i} d_{i y}\right)+c\left(P_{i z}+t_{i} d_{i z}\right)-d=0, \\
& t_{i}=\frac{-\left(a P_{i x}+b P_{i y}+c P_{i z}-d\right)}{a d_{i x}+b d_{i y}+c d_{i z}} .
\end{aligned}
$$

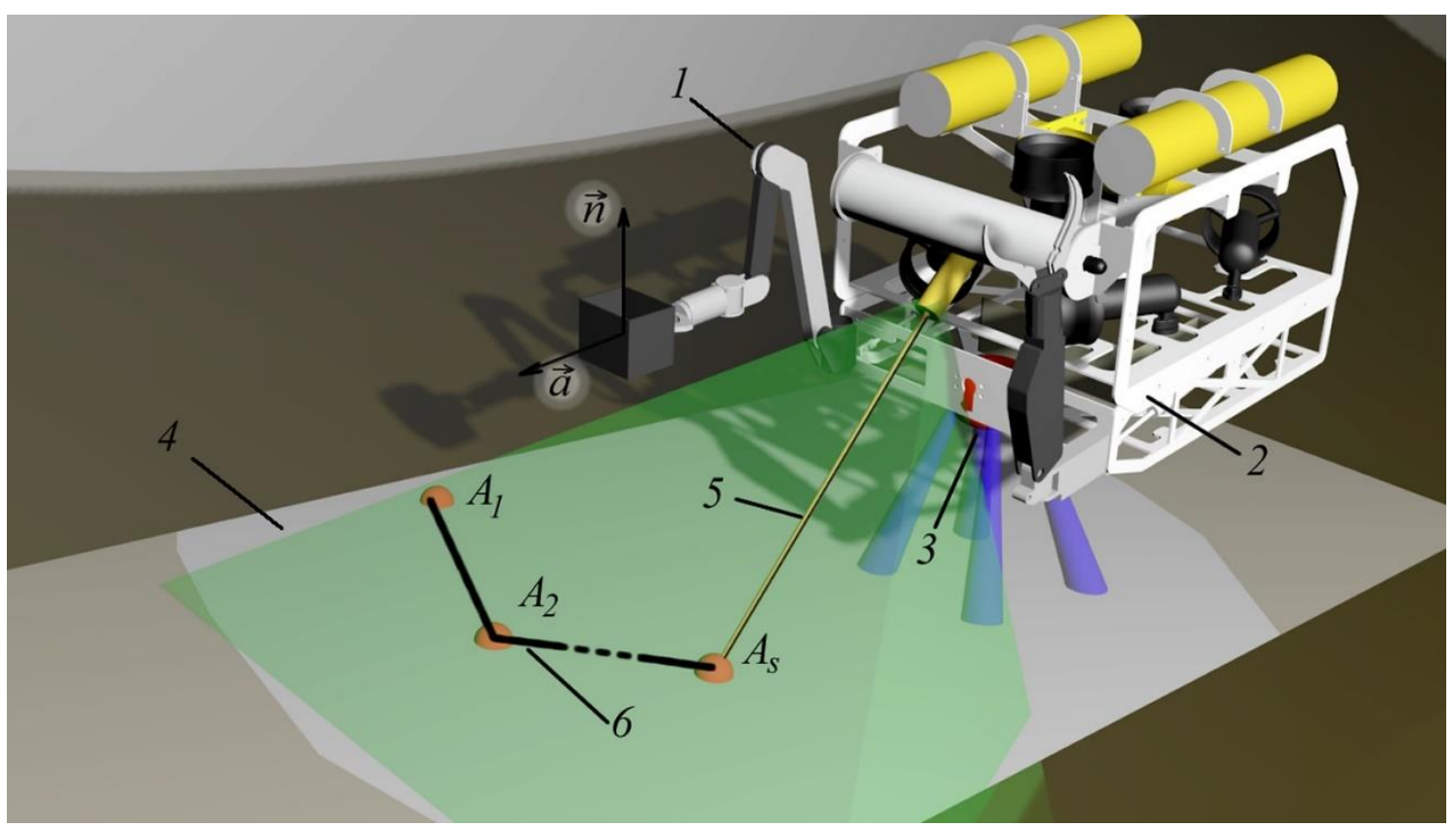

Fig. 2. The performing of an operation in supervisory mode

The coordinates of the point $A_{i}$ of the intersection of the optical axis of the camera with the bottom plane are determined by the substitution of the found value of the parameter $t_{i}$ into the equation (2):

$$
A_{i}=P_{i}+\frac{-\left(a P_{i x}+b P_{i y}+c P_{i z}-d\right)}{a d_{i x}+b d_{i y}+c d_{i z}} \vec{d}_{i}
$$

After the operator directs the optical axis of the camera to the next target point $A_{i+1}$, the value of the parameter $t_{i+l}$ is calculated using the equation (3) for the new point $P_{i+l}$ and the vector $\vec{d}_{i+1}$. The coordinates of the remaining target points are determined in a similar way. As a result, the trajectory of the MM working tool is represented as its successive transition across the all target points.

\section{Formation of the motion trajectory of the MM working tool in a complex bottom relief}

If the bottom surface has a complex shape, then it is necessary to use the multi-beam 3D sonars [8, 9]. These sonars allow to accurately form the clouds of points, which belong to the bottom surface, in the work area of the UV. The triangulation model (see Fig. 3) is formed using these clouds and the known methods [10, 11]. This model is the set of triangular plates connected together. The motion trajectory of the MM working tool is formed on the basis of this model. The trajectory passes through the $A_{i}$ target points, which specified by the operator, on the complex bottom relief. Each $k$ th triangle in the triangulation is given in the CS $x y z$ by the coordinates of three vertices $V_{k 0}, V_{k 1}, V_{k 2} \in R^{3}$, where $k=\overline{1, g}$, $g$ - number of the triangles.

To find the coordinates of each target point $A_{i}$ at the intersection of the optical axis of the camera and the resulting triangulation surface, it is necessary to determine which of the $g$ triangles are intersected by the axis (2) at the current 
time. For this, the Moller-Trumbor algorithm [12] is used, which allows to determine the $k$-th triangle which is intersected by the axis (2). This algorithm uses the information about the coordinates of the point $P_{i}$, the elements of the vector $\vec{d}_{i}$, and also the coordinates of the vertices $V_{k 0}, V_{k 1}, V_{k 2}$ of the all $g$ triangles. The coordinates of the point $A_{i}$ in the CS $x y z$ with respect to the vertices of the $k$-th triangle can be defined in vector form as.

$$
A_{i k}=w_{i k} V_{k 0}+u_{i k} V_{k 1}+v_{i k} V_{k 2} \text {, }
$$

where $u_{i k}, v_{i k}, w_{i k}$ are the barycentric coordinates of the point $A_{i}$ with respect to the vertices of the $k$-th triangle $\left(u_{i k}+v_{i k}\right.$ $\left.+w_{i k}=1\right)$.

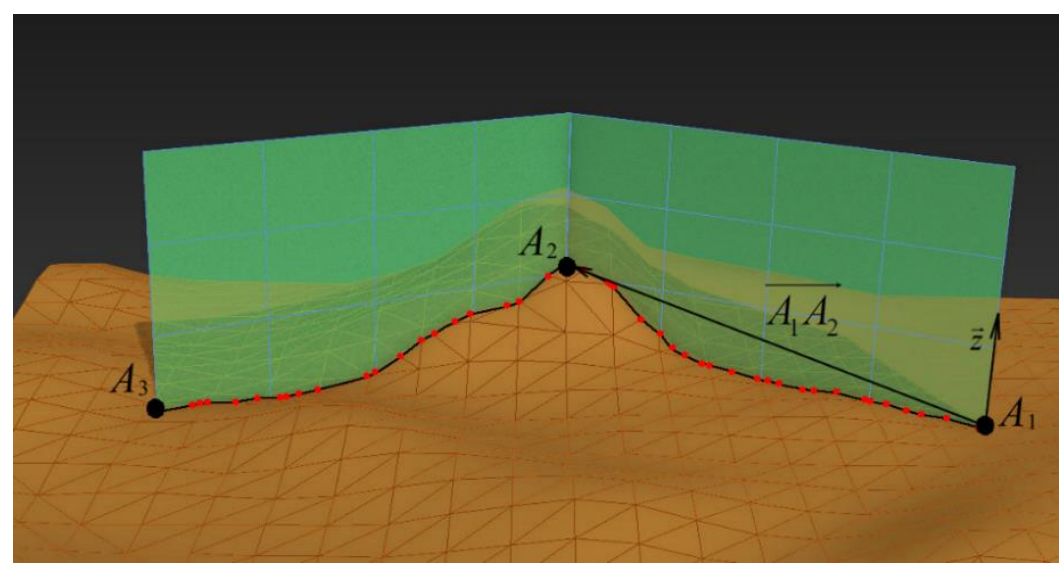

Fig. 3. Triangulation model of the bottom surface

After the calculation of $w_{i k}=1-\left(u_{i k}+v_{i k}\right)$ and the substituting of the axis equation (2) into the equation (4) we obtain a system of equations with the parameters $t_{i k}, u_{i k}, v_{i k}$

$$
P_{i}+t_{i k} \vec{d}_{i}=\left(1-\left(u_{i k}+v_{i k}\right)\right) V_{k 0}+u_{i k} V_{k 1}+v_{i k} V_{k 2}
$$

which can be written in a matrix form

$$
\left[\begin{array}{lll}
-\vec{d}_{i} & V_{k 1}-V_{k 0} & V_{k 2}-V_{k 0}
\end{array}\right]\left[\begin{array}{c}
t_{i k} \\
u_{i k} \\
v_{i k}
\end{array}\right]=\left[P_{i}-V_{k 0}\right]
$$

The values of the parameters $t_{i k}, u_{i k}, v_{i k}$ are found by the solving of the system of equations (5) [12]:

$$
\left[\begin{array}{c}
t_{i k} \\
u_{i k} \\
v_{i k}
\end{array}\right]=\frac{1}{\left(\vec{d}_{i} \times\left(V_{k 2}-V_{k 0}\right)\right) \cdot\left(V_{k 1}-V_{k 0}\right)}\left[\begin{array}{c}
\left(\left(P_{i}-V_{k 0}\right) \times\left(V_{k 1}-V_{k 0}\right)\right) \cdot\left(V_{k 2}-V_{k 0}\right) \\
\left(\vec{d}_{i} \times\left(V_{k 2}-V_{k 0}\right)\right) \cdot\left(P_{i}-V_{k 0}\right) \\
\left(\left(P_{i}-V_{k 0}\right) \times\left(V_{k 1}-V_{k 0}\right)\right) \cdot \vec{d}_{i}
\end{array}\right],
$$

where $(\cdot)$ is the dot product of vectors; $(\times)$ is the cross product of vectors.

As a result, the triangle $k$ with the vertices $V_{k 0}, V_{k 1}$ and $V_{k 2}$, which intersects the axis (2), is found from the equations [12]

$$
\begin{aligned}
& 0 \leq u_{i k} \leq 1, \\
& 0 \leq v_{i k} \leq 1, \\
& u_{i k}+v_{i k} \leq 1 .
\end{aligned}
$$

The coordinates of the point $A_{i}$ of the intersection of the optical axis of the camera with the found triangle $k$ are determined by the substituting of the found parameter $t_{i k}$ from the formula (6) into the equation (2):

$$
A_{i}=P_{i}+\frac{\left(\left(P_{i}-V_{k 0}\right) \times\left(V_{k 1}-V_{k 0}\right)\right) \cdot\left(V_{k 2}-V_{k 0}\right)}{\left(\vec{d}_{i} \times\left(V_{k 2}-V_{k 0}\right)\right) \cdot\left(V_{k 1}-V_{k 0}\right)} .
$$


In order to create the MM working tool trajectory passing through the all the obtained points $A_{i}$ this trajectory is represented as sequence of the triangulation surface cuts. These cuts are the vertical profiles of parts of this surface between the neighboring target points. These profiles can be represented in the form of a set of intersection points of the edges of the triangles in the triangulation and the plane formed by the vector $\overrightarrow{A_{i} A_{i+1}}$, which connects the neighboring target points, and the unit vector $\vec{z}$, which is parallel to the $z$ axis of the CS $x y z$ (see Fig. 3).

For the creation of the MM working tool trajectory, which has to pass through the intersection points of this plane and triangles, it is necessary to calculate which of the all $g$ triangles are intersected by the plane. If the $k$-th triangle and the plane formed by the vectors $\overrightarrow{A_{i} A_{i+1}}$ and $\vec{z}$ intersect, then one triangle vertex will be on the opposite side of the plane than the other two. According to algorithm [7], the computing of the signed distance $p_{i k h}$ between each of $h$-th vertex ( $h=0$, $1,2)$ and this plane and comparing their signs can immediately determine if an intersection exists. The values $p_{i k h}$ are calculated by the formula $p_{i k h}=\vec{r}_{i} \cdot \overrightarrow{A_{i} V_{k h}}, \quad \vec{r}_{i}=\frac{\overrightarrow{A_{i} A_{i+1}} \times \vec{z}}{\left\|\overrightarrow{A_{i} A_{i+1}} \times \vec{z}\right\|}$, where $\overrightarrow{A_{i} V_{k h}}$ is the vector connecting the target point $A_{i}$ and the $h$-th vertex $V_{k h}$ of the $k$-th triangle.

If for any pair of the vertices of the triangle $k$ the corresponding values $p_{i k h}$ have the same signs $\left(p_{i k 0} p_{i k 1}>0\right.$ and $p_{i k 0} p_{i k 2}>0$ or $p_{i k 0} p_{i k 1}<0$ and $\left.p_{i k 0} p_{i k 2}<0\right)$, then the plane does not intersect the $k$-th triangle. If $p_{i k h}$ has different signs $\left(p_{i k 0} p_{i k 1}>0\right.$ and $p_{i k 0} p_{i k 2}<0$ or $p_{i k 0} p_{i k 1}<0$ and $\left.p_{i k 0} p_{i k 2}>0\right)$, then the point $T_{i k e}$ lies on the edge of the triangle $k$, which is formed by this pair of vertices ( $e=1,2$, because in the general case the edges of each triangle can't have more than two points of intersection with the plane). The MM working tool must pass through this point. The coordinates of the point $T_{i k e}$ for the edge formed by the vertices $V_{k 0}$ and $V_{k 1}$ are determined by the formula [7]: $T_{i k e}=V_{k 0}+t_{i k e} \frac{\overrightarrow{V_{k 0} V_{k 1}}}{\left\|\overrightarrow{V_{k 0} V_{k 1}}\right\|}$, where $t_{i k e}=\frac{-\left(\vec{r}_{i} \cdot \overrightarrow{O V_{k 0}}+\left|\overrightarrow{O A_{i}} \cdot \vec{r}_{i}\right|\right)}{\vec{r}_{i} \cdot \overrightarrow{V_{k 0} V_{k 1}}}, \overrightarrow{V_{k 0} V_{k 1}}$ is the vector connecting the vertices $V_{k 0}$ and $V_{k 1}$ of the triangle $k[7],\left|\overrightarrow{O A_{i}} \cdot \vec{r}_{i}\right|-$ the distance from the plane, which formed by the vectors $\overrightarrow{A_{i} A_{i+1}}$ and $\vec{z}$, to the initial point $O$ of the CS $x y z$.

The coordinates of the second point $T_{i k e}$, which lies on the one of the remaining two edges formed by the vertices $V_{k 0}$ and $V_{k 2}$ or $V_{k 1}$ and $V_{k 2}$ of the $k$-th triangle, are calculated similarly. If $p_{i k h}=0$, then the $h$-th vertex of the $k$-th triangle lies on the plane. In this case $T_{i k e}=V_{k h}$.

As a result, the set of points $T_{i k e}$ through which the MM needs to move the working tool (in particular, the soil sampling box) are formed. Moreover, the vector $\vec{a}_{i k}$ in the points $T_{i k 1}$ and $T_{i k 2}$ will always be perpendicular to the plane of the $k$ th triangle, and also when the MM working tool is moving between these points. Therefore, the vector $\vec{a}_{i k}$ in the CS $x y z$ is always perpendicular to the plane defined by the three vertices of the $k$-th triangle.

The resulting set of points $T_{i k e}$ with the corresponding vectors $\vec{a}_{i k}$ is sorted into the sequence of points $T_{j}$ with the vectors $\vec{a}_{j}$ for the creation of the MM motion trajectory. The points $T_{j}$ are located between the neighboring target points $A_{i}$ and $A_{i+1}$, where $j$ is the sequence number of the point in the moving sequence of the MM working tool. The vector $\vec{n}_{j}$ of the working tool is always parallel to the vector $\overrightarrow{T_{j} T_{j+1}}$ connecting the passed and the next points of the sequence.

As a result, the obtained sequence of points $T_{j}$ and corresponding vectors $\vec{a}_{j}$ and $\vec{n}_{j}$ forms the trajectory of the working tool movement, and also can set the different modes of this motion [13].

\section{Program implementation and investigation of the supervisory control method}

The developed method of supervisory control of the MM is implemented in the $\mathrm{C}++$ programming language. The input data for the created program is the set of points belonging to the bottom surface and obtained via hydroacoustic sonars. These points are determined in three-dimensional space (see Fig. 4a). This set is loaded into the program as a text file. The coordinates of the points $P_{i}$ and the vectors $\vec{d}_{i}$ which determine the location of the optical axis of the camera in the CS $x y z$ are recorded in this program.

The developed program builds a triangulation model of the bottom surface (see Fig. 4b). This program makes it using the algorithm [10] implemented in the Point Cloud Library [11]. After the operator sets the points $P_{i}$ and the vectors $\vec{d}_{i}$, the program calculates the coordinates of the target points $A_{i}$, and also forms the sequence of points $T_{j}$ (see Fig. 4b, 4c), and their corresponding vectors $\vec{a}_{j}$ and $\vec{n}_{j}$ of the MM working tool. The visualization of the results of the created program was performed using the $3 \mathrm{~d}$ s Max software product and the ParaView graphic software package. 


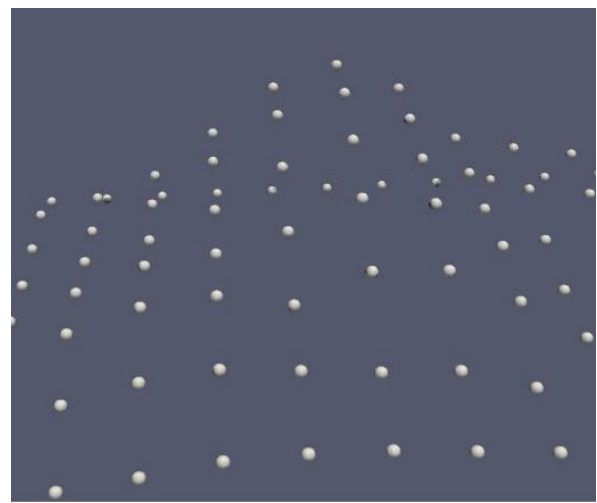

a)

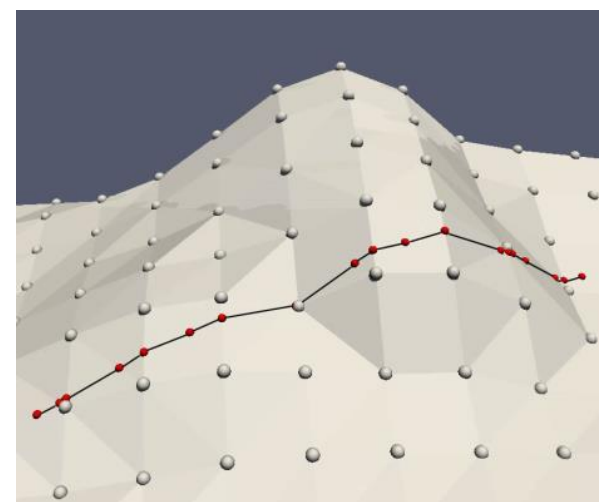

b)

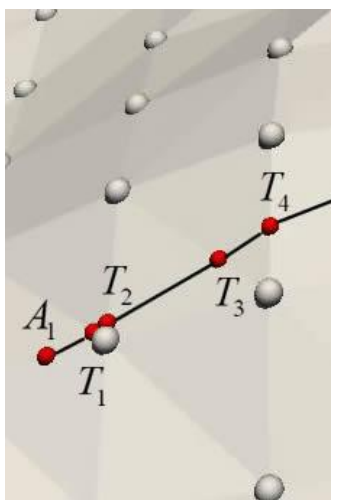

c)

Fig. 4. a) set of points of the bottom surface in the CS $x y z$, b) triangulation model of the bottom and the formed trajectory of the MM working tool, c) detailed description (presentation) of the trajectory

\section{Conclusion}

The underwater technological and research manipulation operations are still performed only in a manual mode by the specially trained operators of the UVs. But quick and accurate performing of the manipulation operations is very difficult for the operator. This leads to the performance decreasing of his work and to the probability increasing of errors.

The developed method can be used to perform many underwater manipulation operations in the supervisory mode. The trajectories and orientation of the MM working tools in the process of motion are formed on the basis of the mathematical model of the work object and taking into account the requirements for the performing of specific technological operations. The developed method is implemented like a program that allows to build triangulation models of the seabed surface and calculate the spatial motion trajectories of the MM working tools.

The future research will include the creation of models of working objects based on the information from the technical vision systems, and the formation of smooth spatial trajectories of the MM working tools. The control systems will be created based on the developed method. These systems will be introduced into the underwater robotic complex, which will be used to perform real technological and research underwater operations.

\section{Acknowledgments}

This work was performed in Institute of Marine Technology Problems FEB RAS, Institute for Automation and Control Processes FEB RAS and also in Far Eastern Federal University (Vladivostok, Russia).

The development and software implementation of the method for the supervisory performing of manipulation operations was carried out with the financial support of the Russian Science Foundation (RSF) grant (project №17-7910064). The theoretical and experimental works on the construction of mathematical models of the bottom surface was carried out with the financial support of the Russian Foundation for Basic Research (RFBR) grants (projects 16-29-04195 Ofi_m, 17-57-45055 Ind_a).

\section{References}

[1] Dubrovin F.S., Scherbatyuk A.F. (2015). Development of Algorithms for an Autonomous Underwater Vehicle Navigation with a Single Mobile Beacon: The Results of Simulations and Marine Trials // Proc. of the XXIIth International Conference on Integrated Navigation Systems, ICINS 2015, Saint Petersburg, Russia. P. $144-152$. ISBN 978-5-91995-023-3.

[2] Filaretov V.F., Konoplin A.Yu. (2015). System of Automatically Correction of Program Trajectory of Motion of Multilink Manipulator Installed on Underwater Vehicle. Procedia Engineering, Vol. 100, 2015. pp. 1441-1449.

[3] Penalver A., Perez J., Fernandez J.J., Sales J., Sanz P.J., Garcia J.C., Fornas D., Marin R. (2015). Visually-guided manipulation techniques for robotic autonomous underwater panel interventions. Annual Reviews in Control 40, pp. 201-211, 2015.

[4] Filaretov V.F., Konoplin A.Yu., Konoplin N.Yu., Gorbachev G.V. (2016). Control system for underwater vehicle with multilink manipulator for automatic manipulation operations, Proceedings of the 27th DAAAM International Symposium, B. Katalinic (Ed.), Published by DAAAM International, Vienna, Austria, 2016, - pp. 714-720. DOI: 10.2507/27th.daaam.proceedings.103.

[5] Korn G.A., Korn T.M. (2000). Mathematical Handbook for Scientists and Engineers: Definitions, Theorems, and Formulas for Reference and Review. General Publishing Company, 2000. - 1151 p. 
[6] Filaretov V.F., Konoplin A.Yu., Konoplin N.Yu. (2017). System for automatically performing of manipulation operations with help of underwater robot. Mechatronics, automation, control. 2017. Iss. 8. - pp. 543-549. (Филаретов В. Ф., Коноплин А.Ю., Коноплин Н.Ю. Система для автоматического выполнения манипуляционных операций с помощью подводного робота // Мехатроника, автоматизация, управление. 2017. №8. T. 18. C. 543-549.)

[7] Schneider, P.J., and Eberly, D.H. (2002) Geometric Tools for Computer Graphics, Elsevier Science Inc., New York.

[8] Tritech Eclipse. Multibeam Sonar for 3D Model View of Sonar Imagery http://www.tritech.co.uk/

[9] BlueView 3D Multibeam Scanning Sonar http://www.teledynemarine.com/

[10] Marton Z., Rusu R., Beetz M. (2009). On Fast Surface Reconstruction Methods for Large and Noisy Datasets" Proceedings of the IEEE International Conference on Robotics and Automation (ICRA), 2009, Kobe, Japan, pp. 3218-3223.

[11] Point Cloud Library: Fast triangulation of unordered point clouds http://ns50.pointclouds.org/

[12] Möller T. and Trumbore B. (1997). Fast, Minimum Storage Ray-Triangle Intersection. Journal of Graphics Tools (JGT), 2(1):21-28, October 1997. 254.

[13] Filaretov V.F., Yukhimets D.A., Konoplin A.Yu. (2014). Synthesis of System for Automatic Formation of Multilink Manipulator Velocity// The Second RSI International Conference on Robotics and Mechatronics (ICRoM 2014). Tehran IRAN. International IEEE Conference. 2014. pp. 785-790. DOI: 10.1109/ICRoM.2014.6990999. 